\title{
Strategic Complements, Substitutes and Ambiguity: The Implications for Public Goods ${ }^{1}$
}

\author{
Jürgen Eichberger \\ Alfred Weber Institut, \\ Universität Heidelberg, Germany \\ David Kelsey \\ Department of Economics, \\ The University of Birmingham.
}

September 1, 2015

Running Title Public Goods and Ambiguity

Address for Correspondence: David Kelsey, Department of Economics, The University of Birmingham, Birmingham, B15 2TT, ENGLAND. tel. +44-121-414-6653, fax. +44-121-414-7377, e-mail D.Kelsey@bham.ac.uk.

2

1 Research supported by ESRC senior research fellowship, award no. H52427502595, ESRC grant no. R000222597 and a grant from the School of Social Science at The University of Birmingham. For comments and discussion we would like to thank Richard Cornes, John Fender, Max Fry, Simon Gächter, Simon Grant, Stephen King, Frank Milne, Sujoy Mukerji, Shasikanta Nandeibam, Yew-Kwang Ng, Thomas Renstrom, Martin Sefton, Peter Wakker and participants in seminars at the Universities of Bielefeld, Birmingham, Bonn, Essex, Hull, Humboldt (Berlin), Kiel, Mannheim, Nottingham, Penn. State, Queens, St. Andrews, Saarlandes, SMU, VPI, York, Birkbeck College, London, the Econometric society meetings in Iowa City 1996, the Warwick summer workshop 1997 and the European Economic Association Meetings 1997, The 2 Public Choice Society meetings 2000, the referee and associate editor of this journal. 


\section{INTRODUCTION}

This paper is concerned with ambiguity (also known as Knightian uncertainty). Ambiguity refers to situations in which individuals have to make decisions when the relevant probabilities are unknown. We believe that many important economic decisions are affected by ambiguity. Our main results concern symmetric $n$-player games with aggregate externalities. We show that ambiguity has different effects depending on whether there are strategic complements or substitutes. As an application, we show that ambiguity will increase voluntary donations to a public good.

\subsection{Background to the Public Goods Model}

Conventional models of public goods imply that voluntary provision is virtually impossible in large societies, due to free-rider problems. Despite this, in practice some public goods are privately provided. For instance, in the UK the National Trust (a private charity) preserves buildings and landscapes and receives a large proportion of its funds from voluntary donations. In the USA, many non-commercial radio and television stations are similarly funded. (Other researchers have also noted that experience does not appear to confirm the theory of free-riding, see e.g. [24], p. 113.) Experimental research has similarly found significantly less free riding than is predicted. It has been usual to explain this by postulating that subjects have altruistic preferences, see e.g., [2] or [38]. In the present paper, we advance an alternative explanation based on ambiguity.

If public goods are financed by voluntary donations, individuals are likely to be uncertain about the contributions of others. In a partial equilibrium model, Austen-Smith [4] modelled this as risk (additive uncertainty) and argued that risk-aversion (i.e. concave utility) would increase contributions. However once general equilibrium effects are taken into account, riskaversion is not sufficient to guarantee an increase in contributions. Public good provision will only increase if a certain restriction on the third derivatives of utility, is satisfied, see [22] and [35]. It does not seem unfair to conclude that there is no general reason to expect risk-aversion to increase voluntary contributions to a public good. In the present paper we show that if 
utility is a concave function of contributions, then uncertainty will indeed cause contributions to increase, if doubts about the behaviour of others are modelled as ambiguity rather than risk.

\subsection{Ambiguity}

Ambiguity refers to situations where it is difficult to assign precise probabilities. It has often been argued that this kind of uncertainty is important for economics. In this paper we shall use a model of ambiguity due to Schmeidler [37], which represents individuals' beliefs by capacities (non-additive subjective probabilities). He axiomatises preferences that can be represented by maximising the expected value of utility with respect to a capacity. (The expectation is expressed as a Choquet integral, [6].) This theory will henceforth be referred to as Choquet Expected Utility (CEU). Under some plausible assumptions, this gives rise to preferences which over-weight the worse outcomes of any given option.

We investigate the influence of ambiguity on the provision of public goods. In our model each individual is uncertain about the contributions of others. Apart from this, the model is deterministic. We believe that it is possible that there may be ambiguity, where public goods are voluntarily provided. One reason is that economic models are an imperfect reflection of reality. A motivation for ambiguity is that it models how decision-makers may behave to protect themselves against errors arising from imperfections in the model. This argument is explained in more detail in Mukerji [32].

We find that ambiguity can reduce free-riding by increasing the perceived marginal benefit of contributions, moreover this effect can be quite large. However, the ambiguous equilibrium will typically not be Pareto optimal since, each individual still fails to take into account benefits going to others. The sub-optimality of conventional equilibria arose from precisely this failure. It is only by accident that the perceived increase in marginal benefit due to ambiguity will offset this effect. One can show, by example, that it is possible to have too much or too little of the public good with ambiguity. ${ }^{1}$ Note that this is qualitatively similar to the experimental evidence, which finds sub-optimal provision but significantly less free-riding than would be predicted by conventional theories.

\subsection{The Role of Returns to Scale}

If there are sufficiently strong increasing returns in the production of public goods, then our conclusions are changed. In this case, ambiguity will discourage voluntary contributions. These results are in contrast to the case of decreasing returns to scale, where we find that ambiguity 
can increase contributions. However the underlying logic of the two problems is similar. If the production function is sufficiently convex, ambiguity reduces the perceived contributions of others. Since the marginal product of donations is falling, this reduces the marginal benefit of contributing. If the payoff function is concave in contributions, then lower perceived contributions by others, increases the anticipated marginal benefit of contributing. Thus voluntary provision is more likely to be successful, the greater the concavity of the production and utility functions.

Alternatively, with increasing returns, there are coordination problems in public good provision. A given individual may wish to make a donation if others do so but not otherwise. Hence, there may be multiple equilibria. Ambiguity increases the coordination problem, since individuals are less able to rely on the contributions of others. This is because, with increasing marginal product, your donation will be more valuable if others also make donations. In summary, ambiguity may reduce free-riding but will increase coordination problems. Provision of public goods, may either increase or decrease depending upon the production and utility functions.

We find it suggestive that, when organisations solicit voluntary contributions, they often emphasise uncertainty concerning future provision and worst case outcomes. (How would you feel if your favourite programme was not broadcast?) This accords well with theoretical models of ambiguity. Material soliciting donations rarely mentions other possible motivations such as altruism.

\section{$1.4 \quad$ Strategic Complements and Substitutes}

We derive our result on public goods from a more general model of symmetric games with aggregate externalities, which we present in section 2 . We investigate how ambiguity interacts with the properties of strategic substitutes and complements in games. These concepts were introduced in [5]. In a game with strategic complements (resp. substitutes), if your opponent increases his/her act, the marginal benefit of increasing your own act will increase (resp. decrease). Examples of games with strategic complements and substitutes are respectively Bertrand and Cournot duopolies with linear demand and constant marginal cost. These concepts have proved useful in industrial organisation. They have also been applied in other areas of economics, most noticeably for instance to coordination problems in macroeconomics see e.g. Cooper and John [7] and Cooper [8]. In section 5 we present some examples of appli- 
cations of our results, in particular to models from industrial organisation, bargaining theory and macroeconomics.

Roughly, we find that ambiguity has opposite effects in games of strategic complements and substitutes. However this is complicated by the possibility that strategic complementarity may give rise to multiple equilibria. If there are positive externalities, ambiguity has the effect of increasing the weight that a player places on the lowest act of his/her opponents. If there are strategic complements (resp. substitutes), this reduces (resp. increases) the marginal benefit of increasing the player's own act and hence the equilibrium strategies. These effects are reversed if there are negative externalities. If there are positive externalities, the Nash equilibrium can be inefficient since players often have an incentive to use strategies below the Pareto optimal level. In this case, for small changes, increasing ambiguity will move the equilibrium towards the symmetric Pareto optimum, if there are strategic substitutes. However, in games with strategic complements, ambiguity will move the equilibrium away from the Pareto optimum.

With strategic complements, it is possible that there might be multiple symmetric equilibria. If there are positive (resp. negative) externalities then higher (resp. lower) equilibria are Pareto superior. In both cases we find that if there is enough ambiguity only the Pareto inferior equilibrium will survive. As a rough guide, our results may be summarised by saying that ambiguity is "helpful" in games with strategic substitutes, but tends to have negative effects when there are strategic complements.

We focus on symmetric equilibria since these are standard in the public goods literature, (see e.g., [9], p. 161). This enables our results to be compared to those obtained without ambiguity. The comparison between asymmetric and symmetric equilibria is orthogonal to that between equilibria with and without ambiguity. Hence, we shall not investigate asymmetric games or equilibria in this paper.

Organisation of the paper The next section introduces CEU preferences and applies them to games. General results on comparative statics in games of aggregate externalities are derived in section 3. We apply these results to public good provision in section 4. Other applications are discussed in section 5 and section 6 concludes. The appendix contains the proofs of those results which are not proved in the text. 


\section{FRAMEWORK AND DEFINITIONS}

\subsection{Games}

This paper is mainly concerned with comparative statics in symmetric games with aggregate externalities, (defined below). Consider a symmetric game $\Gamma=\left\langle N,\left(S_{i}\right)\left(u_{i}\right): 1 \leqslant i \leqslant n\right\rangle$ with finite pure strategy sets $S_{i}$ for each player and payoff functions $u_{i}\left(s_{i}, s_{-i}\right)$. Player $i$ has a finite strategy set which, for convenience, we identify with a subset of the integers, $S_{i}=\left\{0,1, \ldots, m^{*}\right\}$, for $i=1, \ldots, n$. The notation, $s_{-i}$, indicates a strategy combination for all players except $i$. The space of all such strategy profiles is denoted by $S_{-i}$. All players have the same utility function $u\left(s_{i}, s_{-i}\right)$, for $i=1, \ldots, n$. In this paper we shall only consider symmetric equilibria.

DEFINITION 2.1 A game, $\Gamma$, is a symmetric game with positive (resp. negative) aggregate externalities if $u_{i}\left(s_{i}, s_{-i}\right)=u\left(s_{i}, f\left(s_{-i}\right)\right)$, for $1 \leqslant i \leqslant n$, where $u$ is increasing (resp. decreasing) in $f$ and $f: S_{-i} \rightarrow \mathbb{R}$ is increasing in all arguments.

This is a separability assumption. It says that a player only cares about a one-dimensional aggregate of his/her opponents' strategies. Note that it does not restrict two player games, hence our analysis can be applied to all such games. Our main example of such a game is that of voluntary contributions to a public good. In this case, an individual's utility only depends on his/her own consumption of the private good and the total contribution. In particular, utility does not depend on how this total is distributed over others.

NOTATION 2.2 Since $S_{-i}$ is finite, we may enumerate the possible values of $f, f_{0}<\ldots<f_{M}$. Since $f$ is assumed to be increasing $f_{0}=f(0, \ldots, 0)$ and $f_{M}=f\left(m^{*}, \ldots, m^{*}\right)$.

NOTATION 2.3 Let $\Delta\left(s_{i}, s_{-i}\right)=u\left(s_{i}, s_{-i}\right)-u\left(s_{i}-1, s_{-i}\right)$, i.e. $\Delta\left(s_{i}, s_{-i}\right)$ denotes the marginal benefit to individual $i$ of increasing his/her action from $s_{i}-1$ to $s_{i}$ when his/her opponents' strategy profile is $s_{-i}$. If $f\left(s_{-i}\right)=f_{r}$, we may write $\Delta\left(s_{i}, f_{r}\right)$ for $\Delta\left(s_{i}, s_{-i}\right)$.

Note that this is the marginal benefit in the absence of ambiguity. We shall describe later how marginal benefit is modified by ambiguity.

DEFINITION 2.4 A game, $\Gamma$, with aggregate externalities is a game of strategic substitutes (resp. complements) if $\Delta\left(s_{i}, f_{r}\right)$ is a strictly decreasing (resp. increasing) function of $r .^{2}$

The following assumption will be a maintained hypothesis throughout the paper, except in section 4.3 . 
ASSUMPTION 2.5 All games, $\Gamma$, are assumed to be concave, by which we mean that for all $i, u_{i}\left(s_{i}, s_{-i}\right)$ is a strictly concave function of $s_{i} \cdot{ }^{3}$

If there are decreasing returns to scale, the public goods game, discussed in section 4 , is a concave game with positive aggregate externalities and strategic substitutes. A Cournot game with linear demand and constant marginal cost is a concave game with strategic substitutes and negative externalities.

\subsection{Ambiguity}

Traditionally game theory assumes that players have expected utility preferences. We wish to model the effects of ambiguity and hence, assume instead, that the players have CEU preferences. Beliefs about opponents' behaviour are represented by capacities. A capacity assigns non-additive weights, which represent beliefs, to subsets of $S_{-i}$. Formally, capacities are defined as follows.

DEFINITION 2.6 A capacity on $S_{-i}$ is a real-valued function $\nu: \mathcal{P}\left(S_{-i}\right) \rightarrow \mathbb{R}$ (where $\mathcal{P}\left(S_{-i}\right)$ denotes the set of all subsets of $\left.S_{-i}\right)$, which satisfies the following properties:
1. $\quad A \subseteq B \Rightarrow \nu(A) \leqslant \nu(B)$;
2. $\nu(\emptyset)=0, \quad \nu\left(S_{-i}\right)=1$.

Below we define a special class of capacities, which will be useful in our analysis.

DEFINITION 2.7 A capacity $\nu$ is called simple if there exists an additive probability $\pi$ on $S_{-i}$ and a real number $\gamma \in[0,1]$ such that for all events $E \varsubsetneqq S_{-i}, \nu(E)=\gamma \pi(E)$.

Simple capacities are contractions of additive probabilities. The probability distribution $\pi$ can be interpreted as a player's assessment of the relative likelihood of events and the parameter $\gamma$, may be interpreted as the his/her confidence in this assessment.

DEFINITION 2.8 A capacity $\nu$ is convex if $\nu(A)+\nu(B) \leqslant \nu(A \cup B)+\nu(A \cap B)$.

If the capacity is convex, CEU preferences tend to overweight bad outcomes hence they may be termed pessimistic or uncertainty-averse. For a convex capacity, it is possible that $\nu(A)+\nu\left(S_{-i} \backslash A\right)<1$, which implies that not all probability mass is allocated to a set and its complement. This expression can be viewed as a measure of the missing probability mass. It is useful to define the following two measures.

DEFINITION 2.9 The maximal (resp. minimal) degree of ambiguity of capacity $\nu$ is defined by:

$$
\lambda(\nu)=1-\min _{A \subseteq S}(\nu(A)+\nu(\neg A)) \quad\left(\text { resp. } \gamma(\nu)=1-\max _{A \subseteq S}(\nu(A)+\nu(\neg A)) .\right.
$$


The maximal and minimal degrees of ambiguity of the simple capacity $\nu=\gamma \pi$, defined above, have the same value, $1-\gamma$. These definitions are adapted from [12]. They are justified epistemically in [32]. The degrees of ambiguity are measures of the deviation from additivity. For an additive probability they are equal to zero, while for complete uncertainty they are equal to one. ${ }^{4}$

We define the support of a capacity to be the smallest set of opponents' strategies with a complement of capacity zero. For discussion of the definition of support, see [13] and [34].

DEFINITION 2.10 The support of capacity $\nu$ is a set $E \subseteq S_{-i}$, such that $\nu\left(S_{-i} \backslash E\right)=0$ and $\nu(F)>0$, for all $F$ such that $S_{-i} \backslash E \varsubsetneqq F$.

A possible objection to this definition, is that states outside the support may not be Savage-null. We believe that this argument is not valid because the concept of a Savage-null set was formulated for expected utility and is not appropriate in the present context. In expected utility if a state has positive probability, it always enters into the evaluation of an option, while if it has zero probability it never enters into this evaluation. With CEU preferences, there is in addition, a third category of states which may or may not have positive weight depending on how they are ranked. In particular, some states only enter into the evaluation if they yield especially bad outcomes. This third category of states can be interpreted as being "infinitely less likely" than those which are always given positive weight, see [13] and [29]. The definition of support is quite stringent and only includes states which always enter into the evaluation. In particular, it does not include states which only count when they yield bad outcomes.

Player $i$ has beliefs about his/her opponents' behaviour, represented by a capacity $\nu_{i}$ on $S_{-i}$. The expected payoff from a strategy $s_{i}$, is expressed as a Choquet integral over $S_{-i}$. Such preferences have been axiomatised by [21], [36] and [37]. We shall now define the Choquet integral.

NOTATION 2.11 Let $\Gamma$ be a symmetric game with aggregate externalities we shall use $H_{r}$ (resp. $\left.L_{r}\right)$ to denote the event $\left\{s_{-i} \in S_{-i}: f\left(s_{-i}\right) \geqslant f_{r}\right\},\left(\operatorname{resp} .\left\{s_{-i} \in S_{-i}: f\left(s_{-i}\right)<f_{r}\right\}\right)$. Note that $H_{r}=\neg L_{r}$. 
DEFINITION 2.12 Let $\Gamma$ be a symmetric game with positive (resp. negative) aggregate externalities. The Choquet integral of $u_{i}\left(s_{i}, s_{-i}\right)$ with respect to capacity $\nu_{i}$ on $S_{-i}$ is:

$$
\begin{aligned}
V_{i}\left(s_{i}\right) & =\int u_{i}\left(s_{i}, s_{-i}\right) d \nu_{i}=u_{i}\left(s_{i}, f_{M}\right) \nu_{i}\left(H_{M}\right)+\sum_{r=0}^{M-1} u_{i}\left(s_{i}, f_{r}\right)\left[\nu_{i}\left(H_{r}\right)-\nu_{i}\left(H_{r+1}\right)\right] \\
\left(\operatorname{resp} . V_{i}\left(s_{i}\right)\right. & \left.=\int u_{i}\left(s_{i}, s_{-i}\right) d \nu_{i}=u_{i}\left(s_{i}, f_{0}\right) \nu_{i}\left(L_{0}\right)+\sum_{r=1}^{M} u_{i}\left(s_{i}, f_{r}\right)\left[\nu_{i}\left(L_{r}\right)-\nu_{i}\left(L_{r-1}\right)\right]\right) .
\end{aligned}
$$

\subsection{Equilibrium}

We shall use an equilibrium concept based on that of Dow and Werlang [13], which has been extended in [17]. We assume that players do not randomise but play pure strategies. ${ }^{5}$ An equilibrium is an $n$-tuple of capacities, which describes the beliefs of each player about how his/her opponents will play. In equilibrium we require the support of player $i$ 's beliefs consist of his/her opponents' best responses. In the present paper we shall only consider symmetric games and symmetric equilibria. Below we give a formal definition of equilibrium.

DEFINITION 2.13 Let $\Gamma$ be a symmetric game. A capacity $\nu$ on $S_{-i}$ is a symmetric equilibrium of $\Gamma$, if there exists a support, $\operatorname{supp} \nu$ such that for all $i: 1 \leqslant i \leqslant n, \quad \operatorname{supp} \nu \subseteq R(\nu)^{n-1}$, where $R(\nu)=\operatorname{argmax}_{s_{j} \in S_{j}} \int u\left(s_{j}, s_{-j}\right) d \nu$, is the best response correspondence of a player, given beliefs $\nu$.

In a symmetric equilibrium, the beliefs of all players are represented by the same capacity $\nu$, whose support consists of strategies that are best responses for their opponents. In equilibrium, a player's evaluation of a particular strategy may, in part, depend on strategies of his/her opponents which do not lie in the support. We interpret these as events a player views as unlikely but which cannot be ruled out. This may reflect some doubts (s)he may have about the rationality of the opponents or whether (s)he correctly understands the structure of the game. Although in the present paper we only consider symmetric equilibria, it is relatively easy to extend this solution concept to the non-symmetric case (see [13], [17], and [30]).

DEFINITION 2.14 Let $\Gamma$ be a symmetric game and let $\hat{\nu}$ be a symmetric equilibrium of $\Gamma$, if supp $\hat{\nu}$ contains a single strategy profile we say that it is pure, otherwise we say that it is mixed.

Since players choose pure strategies, we are not able to interpret a mixed equilibrium as a randomisation. In a mixed equilibrium some player $i$ say, will have two or more best responses. The support of other players' beliefs about $i$ 's play, will contain some or all of them. Thus an equilibrium, where the support contains multiple strategy profiles, is an equilibrium 
in beliefs rather than randomisations. We note that even without ambiguity, the concept of equilibrium in beliefs has proved useful, see e.g., [3]. If, in addition, it is required that beliefs are additive and the support consists of a single strategy profile, a symmetric equilibrium is a Nash equilibrium. ${ }^{6}$ To analyse the effects of ambiguity it is helpful to define the marginal benefit under ambiguity.

DEFINITION 2.15 Marginal Benefit Suppose that player $i$ has beliefs described by capacity $\nu$ on $S_{-i}$. Define

$$
\operatorname{MB}\left(s_{i}, \nu\right)=\int u\left(s_{i}, s_{-i}\right) d \nu\left(s_{-i}\right)-\int u\left(s_{i}-1, s_{-i}\right) d \nu\left(s_{-i}\right) .
$$

We interpret $\mathrm{MB}\left(s_{i}, \nu\right)$ as player $i$ 's perceived marginal benefit from increasing his/her strategy from $s_{i}-1$ to $s_{i}$, given that (s)he has beliefs represented by capacity $\nu$. In general this will be different to the marginal benefit without ambiguity defined in Notation 2.3.

NOTATION 2.16 If $1 \leqslant k \leqslant m^{*}$, we shall use $r(k)$ to denote that value of $r$ which satisfies $f_{r(k)}=f(k, \ldots, k)$.

The following Lemma finds an expression for the marginal benefit with ambiguity.

LEMMA 2.1 Let $\Gamma$ be a game with aggregate externalities, if $\hat{\nu}$ is a symmetric equilibrium,

a. assume there are positive externalities and $\hat{k}$ is the highest equilibrium strategy, then $\operatorname{MB}\left(s_{i}, \hat{\nu}\right)=\sum_{r=0}^{r(\hat{k})} \Delta\left(s_{i}, f_{r}\right)\left[\hat{\nu}\left(H_{r}\right)-\hat{\nu}\left(H_{r+1}\right)\right] ;$

b. assume there are negative externalities and $\hat{k}$ is the lowest equilibrium strategy, $0<\hat{k}<$ $m^{*}$, then $\operatorname{MB}\left(s_{i}, \hat{\nu}\right)=\sum_{r=r(\hat{k})}^{M} \Delta\left(s_{i}, f_{r}\right)\left[\hat{\nu}\left(L_{r+1}\right)-\hat{\nu}\left(L_{r}\right)\right]$.

As can be seen, the form of marginal benefit is different depending on whether there are positive or negative externalities. This is because the Choquet integral depends on how a given player ranks strategy profiles, which might be played by his/her opponents. These rankings are reversed depending on whether there are positive or negative externalities.

THEOREM 2.2 (Existence of Equilibrium) Let $\Gamma$ be a symmetric game of aggregate externalities, then for any degree of ambiguity $\gamma$, there exists a symmetric equilibrium with degree of ambiguity $\gamma$.

Since the proof of the above theorem demonstrates the existence of an equilibrium in simple capacities, the term "degree of ambiguity" in the statement of the theorem can be interpreted as either the maximal or the minimal degree of ambiguity. 
The following Theorem characterises equilibrium with ambiguity. It is essentially a statement of the usual marginal conditions for equilibrium. The only subtlety is that they are stated in terms of the marginal benefit with ambiguity, as defined in Definition 2.15.

THEOREM 2.3 (Characterisation of Equilibrium) Symmetric equilibria with ambiguity of the game $\Gamma$, may be characterised as follows:

a. $\operatorname{MB}(\hat{m}, \hat{\nu}) \geqslant 0 \geqslant \mathrm{MB}(\hat{m}+1, \hat{\nu})$ is a necessary condition for $\hat{\nu}$ to be an equilibrium, in which all play strategy $\hat{m}$; moreover if $\operatorname{supp} \hat{\nu}=\{\hat{m}\}$ this condition is also sufficient;

b. $\operatorname{MB}(1, \hat{\nu}) \leqslant 0$ (resp. $\left.\operatorname{MB}\left(m^{*}, \hat{\nu}\right) \geqslant 0\right)$ is necessary for $\hat{\nu}$ to be a symmetric equilibrium, in which, all play strategy 0 (resp. $\left.m^{*}\right)$; moreover if $\operatorname{supp} \hat{\nu}=\{0\}$ (resp. $\left.\operatorname{supp} \hat{\nu}=\left\{m^{*}\right\}\right)$ this condition is also sufficient;

c. $\operatorname{MB}(\hat{m}+1, \hat{\nu})=0$ is a necessary condition for $\hat{\nu}$ to be a symmetric equilibrium, in which $\{\hat{m}, \hat{m}+1\}$ is the set of best responses, moreover if $\operatorname{supp} \hat{\nu}=\{\hat{m}, \hat{m}+1\}$ this condition is also sufficient.

The main assumption needed for this result is that each player's utility be concave in his/her own strategy. It holds regardless of the nature of the strategic interactions. Indeed it even holds in the absence of aggregate externalities. However these factors will affect the functional form of MB and hence the equilibrium strategies.

\section{COMPARATIVE STATICS}

In this section we investigate the comparative statics of increasing ambiguity in games with aggregate externalities.

DEFINITION 3.1 We say that capacity $\hat{\nu}$ is more ambiguous than $\bar{\nu}$ if for all non-empty $A \varsubsetneqq S_{-i}, \hat{\nu}(A)+\hat{\nu}(\neg A)<\bar{\nu}(A)+\bar{\nu}(\neg A) \cdot{ }^{7}$

The strict inequality is needed to provide unambiguous comparative statics. Note that if $\hat{\nu}$ is more uncertain than $\bar{\nu}$, then both the maximal and minimal degrees of ambiguity of $\hat{\nu}$ are greater than those of $\bar{\nu}$.

\subsection{Strategic Substitutes}

In games with strategic substitutes and positive externalities, increasing ambiguity has the effect of increasing the strategy played in symmetric equilibrium. There will be alternately pure and mixed equilibria with successively higher strategies. The strategy will monotonically increase from that without ambiguity to the equilibrium with complete uncertainty. These conclusions follow from Proposition 3.1. 
PROPOSITION 3.1 Consider a game of positive (resp. negative) aggregate externalities with strategic substitutes. Let $\bar{\nu}$ be a symmetric pure equilibrium in which $\bar{k}$ is the highest strategy played and let $\hat{\nu}$ be a symmetric equilibrium such that $\hat{\nu}$ is more ambiguous than $\bar{\nu}$, if $\hat{k}$ is the highest (resp. lowest) strategy associated with $\hat{\nu}$ then $\hat{k} \geqslant \bar{k},($ resp. $\hat{k} \leqslant \bar{k})$.

The direction of comparative statics is reversed depending on whether there are positive or negative externalities. With positive externalities, increasing ambiguity increases the weight on lower strategies of the opponent. In a game of strategic substitutes this will increase the marginal benefit of a given player's own action and hence increase the equilibrium strategy. If there are negative externalities, increasing ambiguity will increase the weight on the higher valued actions of one's opponent, such strategies, being the least favourable, in this case.

In both cases, starting from a position of no ambiguity, a small increase in ambiguity will usually move the equilibrium in the direction of the first best symmetric optimum. In games with positive (resp. negative) externalities Nash equilibrium strategies are above (resp. below) the Pareto optimal levels. However we can construct examples, where starting from a high initial level of ambiguity, further increases in ambiguity will cause the equilibrium to move away from the Pareto optimum. ${ }^{8}$

\subsection{Strategic Complements}

With strategic complements, the comparative statics of ambiguity are complicated by the possibility of multiple equilibria. Assume that the lowest equilibrium is always played. Then, with low ambiguity, equilibrium will coincide with that without ambiguity. If there are positive externalities, as ambiguity increases, there will be alternately pure and mixed equilibria, in which sucessively lower strategies will be played. The strategy played in symmetric equilibrium will decline monotonically. In the limit, the equilibrium strategy will tend to the level with complete uncertainty.

Assume instead that the highest equilibrium is played. As before the equilibrium strategy will decline monotonically as ambiguity increases. At some point there is a discrete jump from the highest to the lowest equilibrium. After which, the equilibrium strategy monotonically declines until we reach the level with complete uncertainty. As usual, the comparative statics are reversed if there are negative externalities. These conclusions follow from Propositions 3.2, 3.3 and 3.5. 


\subsubsection{Comparative Statics}

Here we consider comparative statics with strategic complements. We shall deal with the possibility of multiple equilibria in a standard way, by investigating how the highest and lowest equilibria change when ambiguity changes. When there are positive externalities, increasing ambiguity will cause players to place greater weight on low strategies of their opponents. In the presence of strategic complements, this lowers the marginal benefit of a player's own action. Hence more ambiguity decreases the equilibrium contribution level. The following result says that with positive aggregate externalities, an increase in ambiguity causes both the highest and lowest equilibrium strategies to decrease.

PROPOSITION 3.2 Let $\Gamma$ be a game of positive aggregate externalities with strategic complements. Assume $\left\langle\gamma^{\prime}, \lambda^{\prime}\right\rangle \leqslant\left\langle\gamma^{*}, \lambda^{*}\right\rangle$. Let $\underline{k}^{\prime}$ (resp. $\bar{k}^{\prime}$ ) denote the lowest (resp. highest) equilibrium strategy when the minimal degree of ambiguity is $\gamma^{\prime}$ and the maximal degree of ambiguity is $\lambda^{\prime}$. Let $\underline{k}^{*}$ (resp. $\bar{k}^{*}$ ) denote the lowest (resp. highest) equilibrium strategy when the minimal degree of ambiguity is $\gamma^{*}$ and the maximal degree of ambiguity is $\lambda^{*}$. Then $\underline{k}^{\prime} \geqslant \underline{k}^{*}$ and $\bar{k}^{\prime} \geqslant \bar{k}^{*}$.

Next we show that with negative externalities the comparative statics are reversed.

PROPOSITION 3.3 Let $\Gamma$ be a game of negative aggregate externalities with strategic complements. Assume $\left\langle\gamma^{\prime}, \lambda^{\prime}\right\rangle \leqslant\left\langle\gamma^{*}, \lambda^{*}\right\rangle$. Let $\underline{k}^{\prime}$ (resp. $\bar{k}^{\prime}$ ) denote the lowest (resp. highest) equilibrium strategy when the minimal degree of ambiguity is $\gamma^{\prime}$ and the maximal degree of ambiguity is $\lambda^{\prime}$. Let $\underline{k}^{*}$ (resp. $\bar{k}^{*}$ ) denote the lowest (resp. highest) equilibrium strategy when the minimal degree of ambiguity is $\gamma^{*}$ and the maximal degree of ambiguity is $\lambda^{*}$. Then $\underline{k}^{\prime} \leqslant \underline{k}^{*}$ and $\bar{k}^{\prime} \leqslant \bar{k}^{*}$.

\subsubsection{Strategic Complements and Multiple Equilibria}

We now study a special class of games where strategic complementarities are sufficiently strong to generate multiple equilibria. This is characterised by the following assumption.

ASSUMPTION 3.2 $\Delta\left(1, f_{0}\right)<0$ and $\Delta\left(m^{*}, f_{M}\right)>0$.

Assumption 3.2 implies there are always at least three Nash equilibria. The two possible corner solutions are Nash equilibria and there is also an interior equilibrium.

PROPOSITION 3.4 Consider a game of aggregate externalities $\Gamma$, which satisfies Assumption 3.2. Without uncertainty, there are at least three symmetric Nash equilibria:

a. $s_{i}=0$, for $1 \leqslant i \leqslant n$,

b. $s_{i}=m^{*}$, for $1 \leqslant i \leqslant n$,

c. a mixed equilibrium in which players randomise between $\hat{k}-1$ and $\hat{k}$, where $\hat{k}$ satisfies $\hat{k}=\min \left\{k: \Delta\left(k, f_{r(k)}\right) \geqslant 0,0 \leqslant k \leqslant m^{*}\right\}$. 
Games satisfying Assumption 3.2 are, in part, co-ordination games. A given player will wish to play one of the extreme strategies, provided that (s)he believes that others act similarly. Although there are multiple Nash equilibria, the following result shows that only the Pareto inferior equilibrium, survives if there are sufficiently high levels of ambiguity.

PROPOSITION 3.5 Consider a game of positive (resp. negative) aggregate externalities with strategic complements. Under Assumption 3.2, there exists $\bar{\lambda}$ such that if the minimal degree of ambiguity is $\lambda \geqslant \bar{\lambda}$, the equilibrium strategies are unique and all players play strategy 0, (resp. $\left.m^{*}\right)$.

\section{FREE RIDING UNDER UNCERTAINTY}

In this section we apply our results to the voluntary provision of public goods. We have chosen a relatively simple model. This enables us to focus on the effects of ambiguity.

\subsection{The Model}

There are $n$ individuals and two goods, a public good $Y$ and a private good $X$. Each has utility function $u_{i}\left(y, x_{i}\right)=w(y)-d x_{i}$, where $y$ denotes the level of public good provision and $x_{i}$ denotes individual $i$ 's contribution to the public good (in terms of private good). ${ }^{9}$ Contributions may only take integer values in the range $0 \leqslant x_{i} \leqslant m^{*}$. Thus each player has a finite set of pure strategies. This assumption enables us to apply the results from section 3. Individuals are assumed to have a sufficiently large endowment that they are able to contribute $m^{*}$. The level of public good provision is given by the production function, $y=F\left(\sum_{i=1}^{n} x_{i}\right)$.

NOTATION 4.1 Define $G: \mathbb{R} \rightarrow \mathbb{R}$ by $G(x)=w(F(x))$. The function $G$, measures the benefit, in utility terms, of contributions to the public good.

The public goods model is a game of aggregate externalities. To see this define $f: S_{-i} \rightarrow$ $\mathbb{R}$ by $f\left(x_{-i}\right)=\sum_{j \neq i} x_{j}$. There are positive externalities, since any given player's utility is raised when his/her opponents donate more.

\subsection{Decreasing Returns to Scale}

In this subsection we shall assume that there are decreasing returns to scale. The precise sense of this is formalised in the following definition.

ASSUMPTION 4.2 (Concavity) The function $G$ is strictly concave and $G(0)=0$. 
Provided $w$ and $F$ are concave, $G$ will be strictly concave if either there is diminishing marginal utility of the public good ( $w$ is strictly concave) or there are decreasing returns to scale ( $F$ is strictly concave). It is possible to allow for increasing returns to scale in production, provided that these are offset by diminishing marginal utility. When $G$ is concave, the public goods model is a concave game with strategic substitutes. Higher contributions of others increase the supply of the public good, which reduces a given player's own marginal utility of the public good and hence the marginal benefit of his/her own contributions. Lemma 2.1 shows that, in equilibrium, the perceived marginal benefit is a weighted average of marginal benefits at a number of contribution levels for the opponents between 0 and the equilibrium level. When $G$ is concave, this raises the perceived marginal benefit and hence the equilibrium contributions compared to the model without ambiguity.

We shall now investigate comparative statics by changing ambiguity, while keeping other factors constant. As the results in section 3 indicate, increases in ambiguity will increase voluntary contributions to a public good. The following proposition establishes an upper bound for the increase in contributions due to ambiguity.

PROPOSITION 4.1 Let $\bar{m}$ denote the lowest strategy played in symmetric Nash equilibrium (without ambiguity) and let $m_{0}$ denote an equilibrium contribution level with complete uncertainty (maximin preferences). Then $m_{0} \geqslant n \bar{m}$.

Consider a family of symmetric equilibria, which are continuously parametrised by a real number $\alpha$, such that $\alpha=0$ corresponds to no ambiguity (additive beliefs) and $\alpha=1$ corresponds to complete uncertainty and if $\hat{\alpha}>\bar{\alpha}$ then $\nu_{\hat{\alpha}}$ is more ambiguous than $\nu_{\bar{\alpha}}$. As $\alpha$ is increased we get alternately pure and "mixed" equilibria with successively higher levels of contributions. Eventually there will be complete uncertainty i.e. $\alpha=1$. In this case all will contribute $n \bar{m}$ units and the total contribution to the public good will be $n^{2} \bar{m}$. If $n$ is large, this illustrates quite spectacularly how uncertainty can increase provision of a public good. The comparative static properties of the model follow from Propositions 3.1 and 4.1.

The comparative statics of ambiguity are illustrated by figures 1 and 2, which assume that there are two players. Each diagram shows the indifference curves of player 1 , in $x_{1}-$ $x_{2}$ space. We illustrate the case where $G$ is exponential. Figure 1 shows the case of no ambiguity. As usual, the indifference curves are U-shaped and the reaction function of player 1 is downward sloping with slope -1 . In figure 2 there is ambiguity. As ambiguity about player 


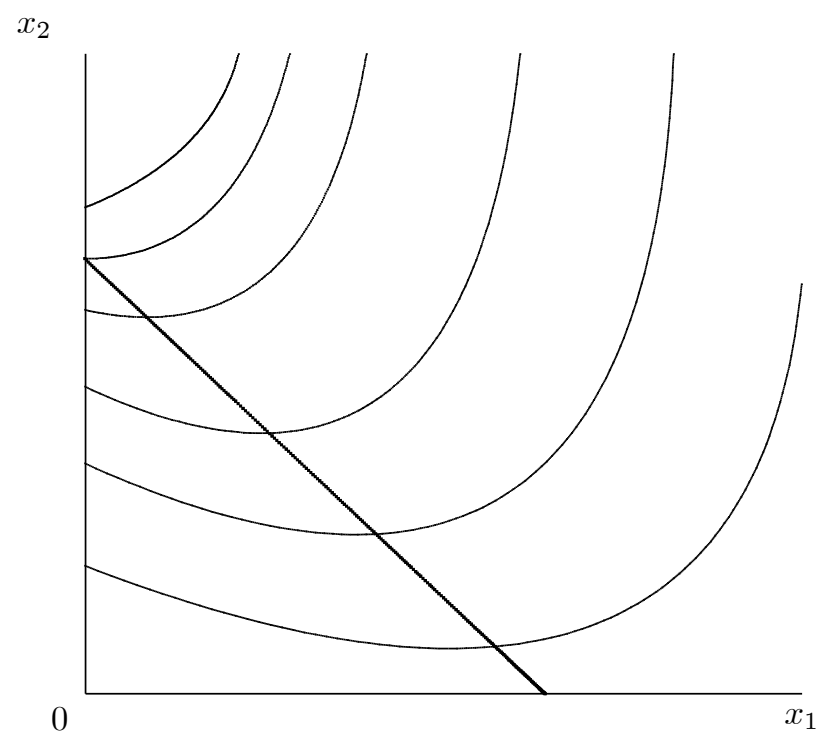

Figure 1: $\gamma=1$

2's contribution increases, it becomes less important to player 1. Thus a unit of player 1's own contribution becomes worth more to him/her in terms of player 2's contributions, which implies the indifference curves become steeper. For similar reasons, changes in player 2's contribution have smaller effects on player 1's optimal contribution, hence player 1's reaction function is steeper when there is ambiguity. The intercepts of the reaction functions remain unchanged while their slopes increase, which implies that equilibrium provision of the public good increases with increases in ambiguity. 


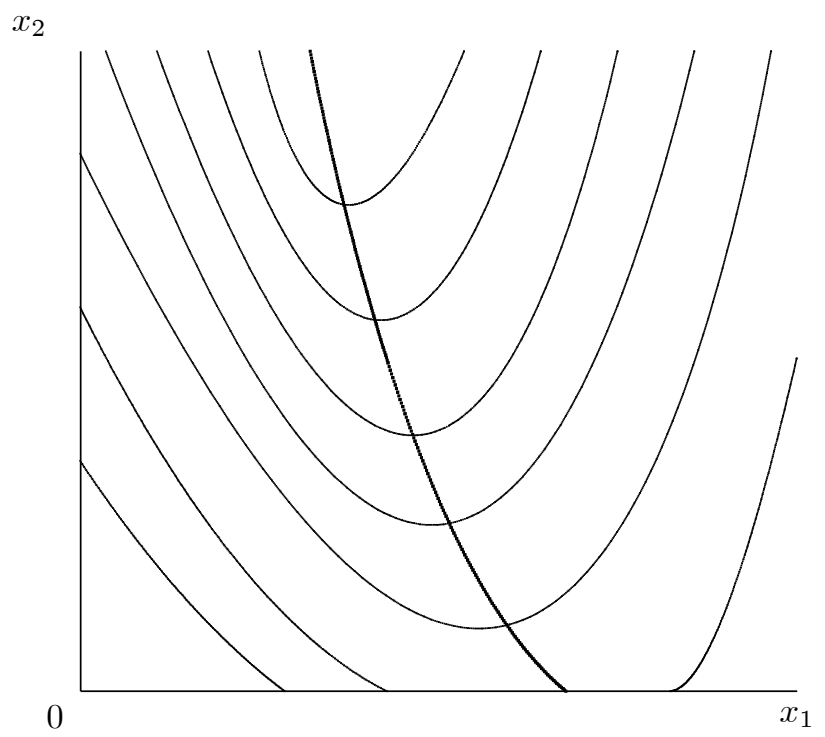

Figure 2: $\gamma=\frac{1}{2}$

\subsection{Increasing Returns to Scale}

In this subsection we investigate how our results are modified if there are increasing returns in the production of public goods. It is possible that the function $G$ will still be concave, if increasing returns in production are offset by diminishing marginal benefit of the public good. In this case the previous results will still apply. If there are sufficiently strong increasing returns to scale to make $G$ convex, ambiguity will reduce public good provision. With convex $G$, marginal benefit is greater, the higher the current level of contributions. When there is ambiguity, any given individual is less able to rely on the contributions of others. Hence ambiguity reduces the anticipated marginal benefit of a contribution. Throughout this subsection we shall require the following assumption to hold.

ASSUMPTION 4.3 (Convexity) $G$ is strictly convex and $G(0)=0$.

NOTATION 4.4 Define $\delta_{r}=G\left(r+m^{*}\right)-G(r)$. Hence $\delta_{r}$ is the extra benefit of contributing $m^{*}$ units rather than 0 , given that the total contribution by other individuals is $r$. Assumption 4.3 implies that $\delta_{r}$ is strictly increasing in $r$.

To set a benchmark, we shall characterise the Nash equilibria (without ambiguity) under Assumption 4.3. There are three possible structures for the set of equilibria, depending on the marginal cost of contributions, $d$. In the first, it is a dominant strategy to make the maximum contribution, $m^{*}$. In the second there are multiple equilibria. Roughly an individual wishes to contribute $m^{*}$ if others do so, but would contribute 0 if (s)he expects others to contribute 
0. In this range, the public goods model is effectively a coordination game. There are two pure strategy equilibria, one of which Pareto dominates the other. Finally, when the marginal cost of contributing is high, it is a dominant strategy for all individuals to contribute 0. Recall $(n-1) m^{*}$, is the largest amount which all individuals, other than $j$, could contribute.

THEOREM 4.2 Characterisation of equilibrium. If there are increasing returns to scale (i.e. Assumption 4.3 is satisfied), symmetric equilibrium without ambiguity may be characterised as follows:

a. if $\delta_{(n-1) m^{*}}>\delta_{0}>m^{*} d$, the only equilibrium contribution level is $m^{*}$, which is a dominant strategy;

b. if $\delta_{(n-1) m^{*}} \geqslant m^{*} d \geqslant \delta_{0}$, there exist two pure strategy equilibria, one in which all contribute 0 and one in which all contribute $m^{*} ;{ }^{10}$

c. if $m^{*} d>\delta_{(n-1) m^{*}}>\delta_{0}$, the only equilibrium contribution level is 0 , which is a dominant strategy.

REMARK 4.1 Since the individual is deciding whether to contribute $m^{*}$ or 0 the marginal cost $d$ of contributing is not relevant. What is relevant is the total cost of contributing which is equal to $m^{*} d$.

Since CEU preferences respect dominance, the two dominant strategy equilibria are unaffected by ambiguity. Hence ambiguity can only have an effect when $\delta_{(n-1) m^{*}} \geqslant m^{*} d \geqslant \delta_{0}$. The following proposition shows that, in almost all cases, where both equilibria exist without ambiguity, only the Pareto inferior equilibrium (without ambiguity) will arise if there is enough ambiguity. This is broadly in line with the experimental results reported in [25].

PROPOSITION 4.3 Assume $\delta_{M} \geqslant m^{*} d>\delta_{0}$, then there exists $\bar{\lambda}$ such that if the minimal degree of ambiguity is $\lambda \geqslant \bar{\lambda}$, the equilibrium strategies are unique and involve all individuals making zero contribution to the public good.

Thus if there are sufficiently strong increasing returns to scale, ambiguity will reduce voluntary donations. When $G$ is concave, increases in ambiguity cause gradual increases in contribution levels. In contrast with convex $G$, increases in ambiguity either have no effect or cause a catastrophic collapse in contributions.

\subsection{Experimental Evidence}

There is a large experimental literature on public goods, for surveys see ( [11], Ch. 6) and [28]. The following stylised facts emerge from this research. 
1. There is no significant evidence of free-riding in single shot games.

2. When subjects play a repeated game, provision of the public good "decays" toward the free-riding level with each repetition.

3. Experienced subjects free-ride more than inexperienced subjects, [24].

4. Unexpectedly changing the rules of the experiment decreases free riding. (This is known as the restart effect, [1].)

5. Face to face communication reduces free-riding, [23].

Intuitively one would expect there to be more ambiguity in one-shot games, the first round of repeated games, with less experienced subjects or after unexpected events. Thus these results seems compatible with our theoretical conclusions. Only the result that face to face communication reduces free-riding fails to support our theory. While communication is likely to reduce uncertainty, it may have other effects such as establishing a focal point, creating feelings of loyalty etc. Hence we do not believe this evidence creates serious problems for our theory, since the reduction in uncertainty is confounded with other factors. The main previous explanation of the observed low level of free riding is altruism. (See, e.g., [2].) However altruism does not explain why repetition decreases free-riding nor the restart effect.

Andreoni [1], investigates the effect of unexpectedly restarting an experiment. Subjects were told that they would play ten rounds of a public goods game. After the tenth round the subjects were told that they would play an additional ten rounds of the same game. The results were that during the first ten rounds, free-riding slowly increased as in other experiments. The first round after restarting had a low level of free-riding similar to first round of the initial experiment. These results appear to be difficult to explain by conventional models of learning, since such models would predict that the first round after the restart would be like the 11th round of a longer experiment. It is possible that the unexpected restart created ambiguity. In which case, the experiment would be compatible with our model. ${ }^{11}$ Andreoni argues that his results can only be explained by "theories of non-standard behaviour". Although he does not explicitly consider Knightian uncertainty, we believe the present paper is in the spirit of this suggestion.

\section{FURTHER APPLICATIONS}

This section suggests some other applications of the results of section 3. In particular we consider Cournot oligopoly, macroeconomic coordination and bargaining. Additional applications to industrial organisation and team production can be found in a companion paper, [16]. 


\subsection{Oligopoly Models}

Cournot oligopoly is a game with aggregate externalities. Consider a model with $n$ firms $1 \leqslant i \leqslant n$. Let $q_{i}$ and $c_{i}\left(q_{i}\right)$ denote respectively the quantity and the cost of firm $i$. Suppose that the inverse demand curve is given by $P(q)$, where $q$ denotes the total quantity supplied. Then the profits of firm $i$ are given by $\pi_{i}=P\left(q_{i}+\sum_{j \neq i} q_{j}\right) q_{i}-c_{i}\left(q_{i}\right)$. If we define $f\left(q_{-i}\right)=\sum_{j \neq i} q_{j}$, this satisfies the requirements of a game of aggregate externalities. Our results imply that total output will be lower in a Cournot oligopoly when there is Knightian uncertainty. This will raise (ex post) profits. However the total of consumer and producer surplus will fall with increases in uncertainty. Hence the effects of ambiguity could be misinterpreted as collusive behaviour by the firms.

\subsection{Bargaining}

We consider a model of bargaining adapted from [33]. There are $n$ individuals $1 \leqslant i \leqslant n$, who bargain over a surplus, $S$, by each claiming a share $s_{i}$ for himself/herself. The rules of bargaining are as follows:

- Each player has a strategy space, $S_{i}$, which consists of the integers between 1 and $t-n+1$, where $t=\alpha n$, for some positive integer $\alpha$. We interpret choosing $s_{i} \in S_{i}$, as claiming a portion $\frac{s_{i} S}{t}$ of the total surplus.

- If the shares claimed by the players are feasible, $\sum_{j=1}^{n} \frac{s_{j}}{t} \leqslant 1$, then they will be implemented.

- If the shares demanded exceed 1 , the payoff is 0 .

Clearly, this is a symmetric game with negative aggregate externalities, since for a given player, only the sum of the opponents' claims matter and a higher demand of the opponents means a lower payoff. Claims are also strategic substitutes because, for low claims of the opponents, the marginal payoff of an extra claim equals this marginal claim, but falls to zero when the claims of others exceed the available surplus. Hence Proposition 3.1 applies.

This game has multiple Nash equilibria. Any set of claims which exactly exhaust the available surplus forms a Nash equilibrium. Since the total surplus is exhausted, the Nash equilibria are efficient. There is a unique symmetric Nash equilibrium, in which all individuals receive fraction $\frac{1}{n}$ of the total surplus. This game has the structure of a coordination game with conflicting interests, similar to the well-known battles of the sexes game.

With sufficient ambiguity, this picture will change. If a player asks for $\frac{S}{t}$, (s)he will receive this regardless of the claims of other players. This gives a higher payoff than if the claims are inconsistent. Being uncertain about his/her opponent's claims, a player may well opt for a low 
claim in order to ensure a positive payoff. If all players reason in this way, they will all bid low and secure their bid. The outcome, however, would be inefficient. Inefficient outcomes are, in fact, commonly observed in bargaining experiments.

For example, consider the case of two players who share a surplus of $S=1$, with claims $s_{i} \in\left\{\frac{3}{4}, \frac{1}{2}, \frac{1}{4}\right\}, S=1$. In this case, the game can be represented in matrix form as follows:

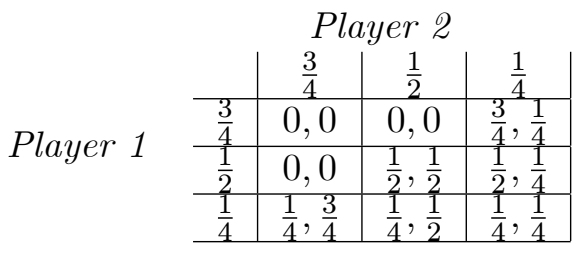

In a symmetric pure equilibrium with beliefs of a typical player represented by capacity $\hat{\nu}$, the possible strategies receive the following (Choquet) expected pay-offs: $V_{1}\left(\frac{3}{4}\right)=\frac{3}{4} \hat{\nu}\left(\frac{1}{4}\right)$; $V_{1}\left(\frac{1}{2}\right)=\frac{1}{2} \hat{\nu}\left(\frac{1}{2}, \frac{1}{4}\right)$ and $V_{1}\left(\frac{1}{4}\right)=\frac{1}{4}$. There are two possible symmetric pure equilibria $\left\langle\frac{1}{2}, \frac{1}{2}\right\rangle$ and $\left\langle\frac{1}{4}, \frac{1}{4}\right\rangle$.

If $\left\langle\frac{1}{2}, \frac{1}{2}\right\rangle$ is a symmetric pure equilibrium with beliefs of a typical player represented by capacity $\hat{\nu}$, then $\hat{\nu}\left(\frac{1}{4}\right)=0$, which implies $V_{1}\left(\frac{3}{4}\right)=0$. For $\frac{1}{2}$ to be a best response it is necessary that $\hat{\nu}\left(\frac{1}{2}, \frac{1}{4}\right) \geqslant \frac{1}{2}$. A sufficient condition for this is that the maximal degree of ambiguity be at most $\frac{1}{2}$.

Now suppose $\left\langle\frac{1}{4}, \frac{1}{4}\right\rangle$ is a symmetric pure equilibrium with beliefs of a typical player represented by capacity $\tilde{\nu}$. For $\frac{1}{4}$ to be a best response it is necessary that $\tilde{\nu}\left(\frac{1}{2}, \frac{1}{4}\right) \leqslant \frac{1}{2}$ and $\tilde{\nu}\left(\frac{1}{4}\right) \leqslant \frac{1}{3}$. A sufficient condition for this is that the minimal degree of ambiguity be at least $\frac{2}{3}$.

Thus increases in ambiguity reduce the equilibrium actions, as predicted by our general results. This is example is interesting. In the Nash equilibrium there are the efficient level of negative externalities. Consequently in equilibria with high minimal degree of ambiguity there are too few negative externalities.

\subsection{Macroeconomic Coordination Games}

There is a large literature on strategic complementarity in macroeconomics, for a survey see Cooper [8]. Our analysis could be used to study the effects of ambiguity in many of these models. As an example we consider a model of social increasing returns to scale based on [8], p. 43. There are $n$ identical individuals. Individual $i$ chooses an effort level $e_{i} \in[0, \bar{e}]$ to maximise his/her utility function $u_{i}=c_{i}-\frac{e_{i}^{2}}{2}$. Each individual produces according to the production function 
$c_{i}=e_{i} \cdot f\left(\sum_{j \neq i} e_{j}\right)$, where $f$ is an increasing function. Thus $u_{i}\left(e_{i}, e_{-i}\right)=e_{i} \cdot f\left(\sum_{j \neq i} e_{j}\right)-\frac{e_{i}^{2}}{2}$. Any given individual $i$ produces under constant returns to scale but due to externalities, there are increasing returns to scale for society as a whole.

This is a game of aggregate externalities, since utility only depends on the total effort exerted by others. Clearly the externalities are positive. One can easily check that $u_{i}\left(e_{i}, e_{-i}\right)$ is a concave function of $e_{i}$, hence this model is a concave game. It is a game of strategic complements, since higher effort by any other individual will increase the marginal product of individual $i$. Thus our results suggest that increases in ambiguity will decease effort and output.

It is possible to construct examples of functions $f$, which give rise to multiple equilibria. In this case, if there is sufficiently high ambiguity, only the lowest equilibrium will survive. ${ }^{12}$ Hence it is possible that a small increase in ambiguity could cause a collapse in economic activity. This process may not be reversible. If starting at a low level equilibrium there is an increase in confidence, the low level equilibrium will continue to exist, hence the economy does not necessarily jump to a higher level of activity, even if there are multiple equilibria with the new level of ambiguity. In practice sudden collapses of business confidence occasionally occur and are often blamed for economic recessions. In contrast business confidence and hence economic booms tend to build up gradually over time. Our model is compatible with these stylised facts.

One could also give a development economics interpretation to this model, in which the players are interpreted as sectors of the economy. It is not unreasonable to postulate that there might be strategic complementarity between investment in different sectors. Thus investment in transport and banking may increase the incentive to invest in industry. The model raises the possibility that there may be multiple equilibria with different levels of economic activity. If there is a low level of confidence in economic institutions then the country may be prevented from reaching the highest equilibrium. In this situation, it would be desirable if the government or the international community could adopt policies which increase confidence. Conversely policies which could create uncertainty or undermine confidence should be avoided.

\section{CONCLUSION}

In this conclusion we would like to comment on the relation between Knightian uncertainty and the technique of introducing "irrational types" in a game of incomplete information. At a 
theoretical level both techniques have the effect of making the equilibrium depend on pay-offs, which would not be achieved in the Nash equilibrium of the underlying game. Some phenomena can be explained by either approach, for instance cooperation in the finitely repeated prisoner's dilemma, (see [13] and [27]).

For many applications we prefer models of Knightian uncertainty, since properties of nonadditive beliefs can, in principle, be tested experimentally. It is not clear to us how one can test which kind of irrational types an individual believes possible. The irrational types approach seems to have difficulty explaining experimental evidence. In an experiment, to hypothesise that subjects believe their opponent may have an irrational type, is close to saying that they believe they are playing a different game to the one the experimenter intended. It seems to weaken the motivation for running controlled experiments if to explain the data, one hypothesises that the subjects do not believe the control. No such difficulties arise, if we explain experimental evidence by the hypothesis that, subject's beliefs can be represented by a convex capacity.

As demonstrated in this paper, in games with Knightian uncertainty, the direction of deviation from Nash equilibrium is determined endogenously by the nature of strategic interaction. In particular it depends on whether a game has strategic complements or substitutes and whether there are positive or negative externalities. These predictions can, in principle, be experimentally tested. In contrast, the irrational types approach seems to make few testable predictions.

There has been much research on non-expected utility theories. For these models to become widely accepted it is important to develop economic applications of them. Many applications have been based on the fact that non-expected utility models can, in some sense, be more risk averse then expected utility preferences. (See the distinction between first order and second order risk aversion in [39].) This paper has presented a new effect. CEU preferences exhibit unambiguous comparative statics in games with strategic substitutes or complements. As indicated by the examples in this paper, we believe this will have a number of economic applications.

\section{Appendix A. Proofs}

This appendix contains the proofs of those results not proved in the main text. Where relevant, we shall only give proofs for games with negative aggregate externalities. The corresponding 
results for games with positive aggregate externalities may be established by similar arguments. The following lemma implies that, in the equilibrium of a concave game, either a single strategy is played or two adjacent strategies are played.

LEMMA A.1 Let $\Gamma$ be a game with positive or negative aggregate externalities. Consider a given player, $i$ say. Let $m_{1}>m_{2}>m_{3}$, be three possible strategies for player $i$. Suppose that with beliefs over $S_{-i}$ given by $\nu, m_{1}$ is indifferent to $m_{3}$, then $m_{2}$ is strictly preferred to $m_{1}$.

Proof. Playing strategy $m_{i}$ yields (Choquet) expected utility: $V\left(m_{i}\right)=\int u\left(m_{i}, s_{-i}\right) d \nu\left(s_{-i}\right)$. There exists $\lambda$ such that $m_{2}=\lambda m_{1}+(1-\lambda) m_{3}$. Since $m_{1}$ and $m_{3}$ are indifferent: $V\left(m_{1}\right)=$ $\lambda V\left(m_{1}\right)+(1-\lambda) V\left(m_{3}\right)=\lambda \int u\left(m_{1}, s_{-i}\right) d \nu\left(s_{-i}\right)+(1-\lambda) \int u\left(m_{3}, s_{-i}\right) d \nu\left(s_{-i}\right)$

$=\int\left[\lambda u\left(m_{1}, s_{-i}\right)+(1-\lambda) u\left(m_{3}, s_{-i}\right)\right] d \nu\left(s_{-i}\right)^{13}<\int u\left(m_{2}, s_{-i}\right) d \nu\left(s_{-i}\right)=V\left(m_{2}\right)$, by strict concavity of $G$. The result follows.

NOTATION A.1 For a given strategy $s_{i} \in S_{i}$, define $m_{i}\left(s_{i}\right)$ to be the smallest element of $\operatorname{argmin}\left\{u_{i}\left(s_{i}, s_{-i}\right): s_{-i} \in S_{-i}\right\}$ i.e. $m_{i}\left(s_{i}\right)$ is the worst strategy which i's opponents could adopt when (s)he chooses strategy $s_{i}$.

LEMMA A.2 Let $\nu=\gamma \pi$ be a simple capacity on $S_{-i}$, where $\pi$ is an additive probability on $S_{-i}$ and $0<\gamma \leqslant 1$, then

a. the support of $\nu$ is unique and $\operatorname{supp} \nu=\operatorname{supp} \pi$;

b. $\int u\left(s_{i}, s_{-i}\right) d \nu\left(s_{-i}\right)=\gamma \mathbf{E}_{\pi} u\left(s_{i}, s_{-i}\right)+(1-\gamma) u\left(s_{i}, m\left(s_{i}\right)\right)$.

Proof. For part a see [17] Lemma 3.3 and for part b see [15], Proposition 3.1.

LEMMA A.3 Let $\Gamma$ be a symmetric game with aggregate externalities, suppose that $\hat{\nu}$ is a symmetric equilibrium:

a. suppose that the equilibrium strategy is $\hat{k}$, then, $\hat{\nu}\left(L_{r}\right)=0$ if $f_{r} \leqslant f(\hat{k}, \ldots, \hat{k}), \hat{\nu}\left(H_{r}\right)=0$ if $f_{r}>f(\hat{k}, \ldots, \hat{k})$;

b. suppose that the equilibrium strategies are $\hat{k}, \hat{k}+1$, then, $\hat{\nu}\left(L_{r}\right)=0$ if $f_{r} \leqslant f(\hat{k}, \ldots, \hat{k})$, $\hat{\nu}\left(H_{r}\right)=0$ if $f_{r}>f(\hat{k}+1, \ldots, \hat{k}+1)$.

Proof. The result holds trivially if $\hat{\nu}(A)=0$, for all $A \varsubsetneqq S_{-i}$. Otherwise for any event $A \subseteq S_{-i}$, $\hat{\nu}(A)>0$, if and only if $\langle\hat{k}, \ldots, \hat{k}\rangle \in A$. The result follows by noting that $\langle\hat{k}, \ldots, \hat{k}\rangle \in H_{r}$ if and only if $f_{r} \leqslant f(\hat{k}, \ldots, \hat{k})$ and $\hat{k} \in L_{r}$ only if $f_{r}>f(\hat{k}, \ldots, \hat{k})$. The proof of part (b) is similar. 
Proof of Lemma 2.1. Consider player $i$. Then $\int u\left(s_{i}, s_{-i}\right) d \nu\left(s_{-i}\right)=u\left(s_{i}, f_{0}\right) \nu\left(L_{1}\right)+$ $\sum_{r=1}^{M} u\left(s_{i}, f_{r}\right)\left[\nu\left(L_{r+1}\right)-\nu\left(L_{r}\right)\right]$. Similarly $\int u\left(s_{i}-1, s_{-i}\right) d \nu\left(s_{-i}\right)=u\left(s_{i}-1, f_{0}\right) \nu\left(L_{1}\right)$ $+\sum_{r=1}^{M} u\left(s_{i}-1, f_{r}\right)\left[\nu\left(L_{r+1}\right)-\nu\left(L_{r}\right)\right]$. By taking the difference of these two expressions, we obtain

$$
\operatorname{MB}\left(s_{i}, \nu\right)=\Delta\left(s_{i}, f_{0}\right) \nu\left(L_{1}\right)+\sum_{r=1}^{M} \Delta\left(s_{i}, f_{r}\right)\left[\nu\left(L_{r+1}\right)-\nu\left(L_{r}\right)\right] .
$$

The result follows from equation ((A-1)) and Lemma A.3.

Proof of Theorem 2.2 Define $\Psi^{i}\left(s_{1}, \ldots, s_{n}\right)=(1-\gamma) u_{i}\left(s_{i}, s_{-i}\right)+\gamma u_{i}\left(s_{i}, m_{i}\left(s_{i}\right)\right)$, for $1 \leqslant i \leqslant n$. Consider the game (without ambiguity) $\Gamma^{\prime}$, where the players have strategy sets $\left\{0,1, \ldots, m^{*}\right\}$ and player $i$ 's utility function is $\Psi^{i}$. This game has a symmetric Nash equilibrium, in which players independently choose a strategy according to the probability distribution $\pi$ on $\left\{0,1, \ldots, m^{*}\right\}$, (see [31] p. 115). This induces a product distribution $\Pi$ on $S_{-i}$.

We assert that the simple capacity $\nu$, defined by $\nu(B)=(1-\gamma) \Pi(B), B \varsubsetneqq S_{-i} ; \nu\left(S_{-i}\right)=$ 1 ; is a symmetric equilibrium of $\Gamma$. Suppose that strategy profile $s^{*}=\left\langle s_{1}^{*}, \ldots, s_{n}^{*}\right\rangle \in S_{-i}$ is in the support of $\nu$, then by Lemma A.2, $\Pi\left(s^{*}\right)>0$, which implies that $\pi\left(s_{j}^{*}\right)>0$, for $j \neq i$. Thus strategy profile $s^{*}$ is given positive probability in the Nash equilibrium of the game $\Gamma^{\prime}$. This implies, $\mathbf{E}_{\Pi} \Psi^{j}\left(s_{j}^{*}, s_{-j}^{*}\right) \geqslant \mathbf{E}_{\Pi} \Psi^{j}\left(s_{j}, s_{-j}^{*}\right)$, for $0 \leqslant s_{j} \leqslant m^{*}$ and $j \neq i$. By Lemma A.2, this is equivalent to $\int u_{i}\left(s_{i}^{*}, s_{-i}^{*}\right) d \nu\left(s_{-i}^{*}\right) \geqslant \int u_{i}\left(s_{i}, s_{-i}^{*}\right) d \nu\left(s_{-i}^{*}\right)$, which establishes that $s_{j}^{*}$ is a best response for $j$, given that his/her beliefs can be represented by capacity $\nu$. It follows that $\nu$ is a symmetric equilibrium with uncertainty.

Proof of Theorem 2.3 Part (a) Consider player 1. Let $\hat{\nu}$ be a symmetric equilibrium which satisfies (a). The expected utilities from strategies $\hat{m}-1, \hat{m}$ and $\hat{m}+1$ units are respectively, $\int u\left(\hat{m}-1, s_{-i}\right) d \hat{\nu}\left(s_{-i}\right), \int u\left(\hat{m}, s_{-i}\right) d \hat{\nu}\left(s_{-i}\right)$ and $\int u\left(\hat{m}+1, s_{-i}\right) d \hat{\nu}\left(s_{-i}\right)$. Thus $\operatorname{MB}(\hat{m}, \hat{\nu}) \geqslant 0 \geqslant \operatorname{MB}(\hat{m}+1, \hat{\nu})$ is a necessary condition for contributing $\hat{m}$ units to be a best response. Concavity of $u$ implies that this condition is also sufficient. Hence, if this condition is satisfied and $\operatorname{supp}(\hat{\nu})=\{\hat{m}\}, \hat{\nu}$ is a symmetric equilibrium in which all contribute $\hat{m}$. Part (b) of the theorem follows by similar reasoning.

Part (c) For $\hat{m}$ and $\hat{m}+1$ to both be best responses the individual must be indifferent between them. Hence $\int u\left(\hat{m}, s_{-i}\right) d \hat{\nu}\left(s_{-i}\right)=\int u\left(\hat{m}+1, s_{-i}\right) d \hat{\nu}\left(s_{-i}\right)$. Rearranging we find $\mathrm{MB}(\hat{m}+1, \hat{\nu})=0$. Concavity implies that this condition is also sufficient for $\hat{m}, \hat{m}+1$ to be best responses. The result follows. 
Proof of Proposition 3.1 Suppose that the result is false, then $\hat{k}>\bar{k}$. If $r=M+1$, then $1=\bar{\nu}\left(L_{r}\right) \geqslant \hat{\nu}\left(L_{r}\right)$. If $r(\bar{k})<r \leqslant M$, then by Lemma A.3, $\bar{\nu}\left(H_{r}\right)=0$, hence $\bar{\nu}\left(L_{r}\right)=$ $\bar{\nu}\left(L_{r}\right)+\bar{\nu}\left(H_{r}\right)>\hat{\nu}\left(L_{r}\right)+\hat{\nu}\left(H_{r}\right) \geqslant \hat{\nu}\left(L_{r}\right)$, where the first inequality follows from the fact that $\hat{\nu}$ is more uncertain than $\bar{\nu}$. If $1 \leqslant r \leqslant r(\hat{k}-1)$, then $\hat{\nu}\left(L_{r}\right)=0$, by Lemma A.3, hence $\bar{\nu}\left(L_{r}\right) \geqslant \hat{\nu}\left(L_{r}\right)$ in this case. The assumption $\hat{k}>\bar{k}$, implies that $\hat{k}-1 \geqslant \bar{k}$. Hence $\bar{\nu}\left(L_{r}\right) \geqslant \hat{\nu}\left(L_{r}\right)$ for all $r: 0 \leqslant r \leqslant M+1$, with strict inequality if $r(\bar{k}) \leqslant r \leqslant M$.

Note that $\operatorname{MB}(\nu, k)$ is a weighted average of the $\Delta$ 's. Since $\sum_{r=t}^{m^{*}}\left[\bar{\nu}\left(L_{r}\right)-\bar{\nu}\left(L_{r-1}\right)\right]=$ $1-\bar{\nu}\left(L_{t-1}\right) \leqslant 1-\hat{\nu}\left(L_{t-1}\right)=\sum_{r=t}^{m^{*}}\left[\hat{\nu}\left(L_{r}\right)-\hat{\nu}\left(L_{r-1}\right)\right]$ for $0<t \leqslant M$, (with some strict inequalities) the weights in the expression for $\mathrm{MB}(\bar{\nu}, k)$ first order stochastically dominate those in the expression for $\operatorname{MB}(\hat{\nu}, k)$. Because $\Delta\left(s_{i}, f_{r}\right)$ is a strictly decreasing function of $s_{-i}$, it follows that $\operatorname{MB}(\bar{\nu}, k)<\operatorname{MB}(\hat{\nu}, k)$, for $0 \leqslant k \leqslant m^{*}$.

By Theorem 2.3, $0 \geqslant \operatorname{MB}(\hat{k}+1, \hat{\nu}) \geqslant \operatorname{MB}(\bar{k}, \hat{\nu})>\operatorname{MB}(\bar{k}, \bar{\nu})$. However, by Theorem 2.3, a necessary condition for $\bar{k}$ (resp. $\bar{k}, \bar{k}+1$ ) to be an equilibrium contribution level (resp. levels) with beliefs $\bar{\nu}$, is $\operatorname{MB}(\bar{k}, \bar{\nu}) \geqslant 0$, (resp. $\operatorname{MB}(\bar{k}, \bar{\nu})=\operatorname{MB}(\bar{k}+1, \bar{\nu})=0)$. This contradiction establishes the result.

LEMMA A.4 Let $\Gamma$ be a game of negative aggregate externalities and strategic complements. Suppose that $(1-\gamma) \Delta\left(\bar{m}, f_{r(\bar{m})}\right)+\gamma \Delta\left(\bar{m}, f_{M}\right) \geqslant 0, \bar{m} \neq m^{*}$, then there exists $k \geqslant \bar{m}$ such that there is a pure symmetric equilibrium in simple capacities with degree of ambiguity $\gamma$ in which strategy $k$ is played.

Proof. Suppose first that $(1-\gamma) \Delta\left(k, f_{r(k)}\right)+\gamma \Delta\left(k, f_{M}\right) \geqslant 0$, for all $k \geqslant \bar{m}$, then by Theorem 2.3, there is a corner equilibrium in simple capacities with degree of ambiguity $\gamma$, in which all play $m^{*}$. Otherwise, let $\tilde{k}+1$ be the first integer greater than $\bar{m}$ such that, $(1-\gamma) \Delta\left(\tilde{k}+1, f_{r(\tilde{k}+1)}\right)+\gamma \Delta\left(\tilde{k}+1, f_{M}\right)<0$. By strategic complementarity this implies,

$$
(1-\gamma) \Delta\left(\tilde{k}+1, f_{r(\tilde{k})}\right)+\gamma \Delta\left(\tilde{k}+1, f_{M}\right)<0 .
$$

Then, by definition of $\tilde{k}$,

$$
(1-\gamma) \Delta\left(\tilde{k}, f_{r(\tilde{k})}\right)+\gamma \Delta\left(\tilde{k}, f_{M}\right) \geqslant 0
$$

Equations (A-2) and (A-3) imply the existence of a pure equilibrium in which all play $\tilde{k}$ and beliefs are represented by simple capacities with degree of ambiguity $\gamma$. Clearly $\tilde{k} \geqslant \bar{m}$. 
LEMMA A.5 Let $\Gamma$ be a game of negative aggregate externalities with strategic complements and let $\tilde{k}$ denote the highest strategy played in a symmetric equilibrium with maximal degree of ambiguity at most $\lambda$. Then there exists an equilibrium in simple capacities with degree of ambiguity $\lambda$, in which $\tilde{k}$ is an equilibrium strategy.

Proof. Let $\tilde{\nu}$ be a symmetric equilibrium with maximal degree of ambiguity at most $\lambda$, in which strategy $\tilde{k}$ is played. By definition $\tilde{k}$ is the highest strategy played in this equilibrium. By Lemma 2.1, $\mathrm{MB}\left(s_{i}, \tilde{\nu}\right)=\sum_{r=r(\hat{k})}^{M} \Delta\left(s_{i}, f_{r}\right)\left[\tilde{\nu}\left(L_{r+1}\right)-\tilde{\nu}\left(L_{r}\right)\right]$. Since there are strategic complements, $\Delta\left(k, f_{M}\right)>\Delta\left(k, f_{r}\right)$, for $r(\tilde{k}) \leqslant r<M$. Hence $\tilde{\nu}\left(L_{r(\tilde{k})+1}\right) \Delta\left(k, f_{r(\tilde{k})}\right)+$ $\left(1-\tilde{\nu}\left(L_{r(\tilde{k})+1}\right)\right) \Delta\left(k, f_{M}\right) \geqslant \operatorname{MB}(k, \tilde{\nu})$. Since $\lambda(\tilde{\nu}) \leqslant \lambda, \tilde{\nu}\left(L_{r(\tilde{k})+1}\right)=\tilde{\nu}\left(L_{r(\tilde{k})+1}\right)+\tilde{\nu}\left(H_{r(\tilde{k})+1}\right) \geqslant$ $1-\lambda$. Hence, $(1-\lambda) \Delta\left(k, f_{r(k)}\right)+\lambda \Delta\left(k, f_{M}\right) \geqslant \operatorname{MB}(\tilde{k}, \tilde{\nu}) \geqslant 0$. This equation implies by Lemma A.4, that there is an equilibrium in simple capacities with degree of ambiguity $\lambda$ in which the equilibrium strategy is $\hat{k} \geqslant \tilde{k}$. Since $\tilde{k}$ is the highest equilibrium strategy when the minimal degree of ambiguity is $\lambda$, we must have $\hat{k}=\tilde{k}$. The result follows.

Proof of Proposition 3.3 By Lemma A.4, since $\bar{k}^{*}$ is the highest equilibrium strategy when the maximal degree of ambiguity is $\lambda^{*},\left(1-\lambda^{*}\right) \Delta\left(k, f_{r(k)}\right)+\lambda^{*} \Delta\left(k, f_{M}\right)<0$, for $\bar{k}^{*}+1 \leqslant$ $k \leqslant m^{*}$. Hence $\left(1-\lambda^{\prime}\right) \Delta\left(k, f_{r(k)}\right)+\lambda^{\prime} \Delta\left(k, f_{M}\right)<0$, for $\bar{k}^{*}+1 \leqslant k \leqslant m^{*}$, which implies there is no equilibrium in simple capacities with degree of ambiguity $\lambda^{\prime}$ in which a strategy $\bar{k}^{*}+1 \leqslant k \leqslant m^{*}$, is played. By Lemma A.5, there is no equilibrium with maximal degree of ambiguity $\lambda^{\prime}$ in which a strategy $k, \bar{k}^{*}+1 \leqslant k \leqslant m^{*}$, is played. Hence $\bar{k}^{\prime} \leqslant \bar{k}^{*}$. By similar reasoning we may show $\underline{k}^{\prime} \leqslant \underline{k}^{*}$.

NOTATION A.2 Define

$$
z_{j}^{(n-1)} \equiv\left\{\begin{array}{cc}
\left(\begin{array}{c}
n-1 \\
j
\end{array}\right) \pi^{j}(1-\pi)^{n-1-j}, & (0 \leqslant j \leqslant n-1) \\
0, & (n \leqslant j) .
\end{array}\right.
$$

Thus $z_{j}^{(n-1)}=\operatorname{Pr}\left(Z_{n-1}=j\right)$, where $Z_{n-1}$ is a random variable with the Binomial distribution, parameters $n-1$ and $\pi$.

Proof of Proposition 3.4 Assumption 3.2 directly implies that cases a and b are Nash equilibria. Next we demonstrate the existence of the equilibrium of part c.

Consider a given player $i$, say. Assume that $i$ 's opponents are independently randomising between $\hat{k}$ and $\hat{k}-1$, playing strategy $\hat{k}$ with probability $\pi$. Since $\Gamma$ is symmetric, the value of $f$ only depends on the number of individuals who play $\hat{k}$. Let $f^{j}$ denote the value of $f$ when $j$ individuals play $\hat{k}$ and $n-j$ individuals play $\hat{k}-1$. If $i$ plays strategy $\hat{k}-1$ (resp. $\hat{k}$ ) (s)he will receive, $\sum_{j=0}^{n-1} z_{j}^{n-1} u\left(\hat{k}-1, f^{j}\right)$, (resp. $\left.\sum_{j=0}^{n-1} z_{j}^{n-1} u\left(\hat{k}, f^{j}\right)\right)$. In a mixed equilibrium these 
must be equal, hence,

$$
\sum_{j=0}^{n-1} z_{j}^{n-1} \Delta\left(\hat{k}, f^{j}\right)=0
$$

If $\pi=0$, the expression in equation (A-4) is $\Delta\left(\hat{k}, f^{0}\right)=\Delta\left(\hat{k}, f_{r(\hat{k}-1)}\right)$. By construction $\Delta\left(\hat{k}-1, f_{r(\hat{k}-1)}\right) \leqslant 0$. If $\pi=1$, then the expression in equation (A-4) is $\Delta\left(\hat{k}, f^{n-1}\right)=$ $\Delta\left(\hat{k}, f_{r(\hat{k})}\right)>0$. Therefore by the intermediate value theorem, there exists $\hat{\pi}$ such that equation (A-4) is satisfied. Since $\Gamma$ is a concave game, this is a sufficient condition for existence of a mixed equilibrium.

Proof of Proposition 3.5 Let $\nu$ be a symmetric equilibrium with minimal degree of ambiguity $\lambda$. Consider a given individual, if (s)he plays strategy $m\left(\right.$ resp. $\left.m^{*}\right)$ (s)he will receive utility $u\left(m, f_{0}\right) \nu\left(L_{1}\right)+\sum_{r=1}^{M} u\left(m, f_{r}\right)\left[\nu\left(L_{r+1}\right)-\nu\left(L_{r}\right)\right]$ (resp. $u\left(m^{*}, f_{0}\right) \nu\left(L_{1}\right)+$ $\left.\sum_{r=1}^{M} u\left(m^{*}, f_{r}\right)\left[\nu\left(L_{r+1}\right)-\nu\left(L_{r}\right)\right]\right)$. Hence the extra utility $j$ gets from playing strategy $m^{*}$ rather than $m$, is $\tilde{\delta}_{0} \nu\left(L_{1}\right)+\sum_{r=1}^{M} \tilde{\delta}_{r}\left[\nu\left(L_{r+1}\right)-\nu\left(L_{r}\right)\right]$, where $\tilde{\delta}_{r}=u\left(m^{*}, f_{r}\right)-u\left(m, f_{r}\right)$. By strategic complementarity, $\tilde{\delta}_{r}$ is increasing in $r$. Hence $(1-\lambda) \tilde{\delta}_{0}+\lambda \tilde{\delta}_{M} \leqslant \tilde{\delta}_{0} \nu\left(L_{1}\right)+$ $\sum_{r=1}^{M} \tilde{\delta}_{r}\left[\nu\left(L_{r+1}\right)-\nu\left(L_{r}\right)\right]$. By Assumption 3.2, $\Delta\left(m^{*}, f_{M}\right)>0$, which together with concavity of $u$ implies $\tilde{\delta}_{M}>0$. Therefore, for $\lambda$ sufficiently large, $(1-\lambda) \tilde{\delta}_{0}+\lambda \tilde{\delta}_{M}>0$, which implies that $m$ is not a best response. It follows that for large minimal degrees of ambiguity, the only equilibrium is where all individuals play strategy $m^{*}$.

Proof of Proposition 4.1 Since $\bar{m}$ is a symmetric Nash equilibrium contribution level, it is a best response for player $i$. A necessary condition for this is $\Delta\left(\bar{m}, \bar{m}_{-i}\right) \geqslant d$, where $\bar{m}_{-i}$ denotes the profile where all of $i$ 's opponents contribute $\bar{m}$. Note that for the public goods model, $\Delta(x, y)=\Delta(x+y, 0)$. Hence $\Delta(n \bar{m}, 0) \geqslant d$. The equilibrium under complete uncertainty, $m_{0}$ satisfies $\Delta\left(m_{0}, 0\right) \geqslant d \geqslant \Delta\left(m_{0}+1,0\right)$. By concavity, $\Delta(x, 0)$ is a decreasing function of $x$, which implies $m_{0} \geqslant n \bar{m}$.

Proof of Theorem 4.2 First note that since $G$ is convex, any given individual's best response to a given level of contribution $r$ by the other players is either 0 or $m^{*}$. Hence, for any set of beliefs about the opponents' behaviour 0 or $m^{*}$ will be optimal.

Case a. Consider a given individual $i$, say. By convexity, it is sufficient to show that 0 is not a best response. In a symmetric equilibrium, there will be some number $\pi$ such that any given individual plays $m^{*}$ with probability $\pi$ and 0 with probability $1-\pi$. Then $\sum_{k=0}^{n-1} z_{k}^{(n-1)} G\left(k m^{*}+m^{*}\right)-m^{*} d$ is the benefit to $i$ of contributing $m^{*} \cdot{ }^{14}$ The benefit of con- 
tributing 0 is given by: $\sum_{k=0}^{n-1} z_{k}^{(n-1)} G\left(k m^{*}\right)$. The difference between the utility from contributing $m^{*}$ and that from contributing 0 is given by: $\sum_{k=0}^{n-1} z_{k}^{(n-1)} \delta_{k m^{*}}-m^{*} d>\delta_{0}-m^{*} d>0$, where the first inequality follows from the fact that the $\delta$ 's are increasing and the second is true by assumption. It follows that contributing $m^{*}$ gives strictly higher utility than 0 and hence $m^{*}$ is the unique best response.

Case b. Suppose now, that $i$ believes that all other individuals will contribute $m^{*}$. Then if $i$ contributes $m^{*}$, (s)he will obtain utility $G\left(n m^{*}\right)-m^{*} d$. If $i$ contributes 0 , (s)he will obtain utility $G\left((n-1) m^{*}\right)$. By assumption $\delta_{(n-1) m^{*}}=G\left(n m^{*}\right)-G\left((n-1) m^{*}\right) \geqslant m^{*} d$, hence a best response for $i$ is to contribute $m^{*}$. Similarly we may show that if all others contribute 0 , it is a best response for $i$ also to contribute 0 .

The proof for case (c) is similar to case (a).

Proof of Proposition 4.3 By Lemma 2.1, MB $\left(s_{i}, \hat{\nu}\right)=\sum_{r=0}^{r(\hat{k})} \Delta\left(s_{i}, f_{r}\right)\left[\hat{\nu}\left(H_{r}\right)-\hat{\nu}\left(H_{r+1}\right)\right]$. Note that the proof of Lemma 2.1 does not use concavity hence it is legitimate to apply it in the present context. Let $\nu$ be a symmetric equilibrium under uncertainty with degree of ambiguity $\lambda$. Let $x_{R}=(n-1) m^{*}$, the largest possible contribution of individuals other than $i$. Define $\tilde{H}_{r}=\left\{x_{-i}: \sum_{j \neq i} x_{j} \geqslant r\right\}$. Consider a given individual $j$, if (s)he contributes 0 (resp. $m$ ) units to the public good, (s)he will receive utility $G(R) \nu\left(\tilde{H}_{R}\right)+\sum_{r=0}^{R-1} G(r)\left[\nu\left(\tilde{H}_{r}\right)-\nu\left(\tilde{H}_{r+1}\right)\right]$, $\left(\right.$ resp. $\left.(G(R+m)-m d) \nu\left(\tilde{H}_{R}\right)+\sum_{r=0}^{M-1}(G(r+m)-m d)\left[\nu\left(\tilde{H}_{r}\right)-\nu\left(\tilde{H}_{r+1}\right)\right]\right)$. Hence the extra utility $j$ gets from contributing $m$ rather than 0 , is $\xi_{R} \nu\left(\tilde{H}_{R}\right)+\sum_{r=0}^{R-1} \xi_{r}\left[\nu\left(\tilde{H}_{r}\right)-\nu\left(\tilde{H}_{r+1}\right)\right]-$ $m d$, where $\xi_{r}=G(r+m)-G(r)$. Since $G$ is convex, $\xi_{r}$ is increasing in $r$, hence $(1-\lambda) \xi_{R}+$ $\lambda \xi_{0} \geqslant \xi_{R} \nu\left(\tilde{H}_{R}\right)+\sum_{r=0}^{R-1} \xi_{r}\left[\nu\left(\tilde{H}_{r}\right)-\nu\left(\tilde{H}_{r+1}\right)\right]$. By assumption, $m^{*} d>\delta_{0}$, which together with convexity of $G$ implies $\xi_{0}<m d$. Therefore, for $\lambda$ sufficiently large, $(1-\lambda) \xi_{M}+\lambda \xi_{0}<m d$, which implies that $m$ is not a best response. It follows that for large degrees of ambiguity, the only equilibrium is where all individuals contribute 0 .

\section{References}

[1] J. Andreoni, Why free ride? J. Public Econ, 37 (1988), 291-304.

[2] J. Andreoni, Cooperation in public goods experiments: Kindness or confusion? Amer. Econ. Rev., 85 (1995), 891-904. 
[3] R. J. Aumann and A. Brandenburger, Epistemic conditions for Nash equilibrium. Econometrica, 63 (1995), 1161-1180.

[4] D. Austen-Smith, Individual contributions to public goods. Econ. Letters, 5 (1980), 359-361.

[5] J. Bulow, J. Geanakopolos, and P. Klemperer, Multimarket oligopoly: Strategic complements and substitutes. J. of Polit. Economy, 93 (1985), 488-511.

[6] G. Choquet, Theory of capacities. Annales Institut Fourier, 5 (1953-4), 131-295.

[7] R. Cooper and A. John, Coordinating coordination failures in Keynesian models. Quart. J. of Econ., 103 (1988), 441-463.

[8] R. W. Cooper, "Coordination Games". Cambridge University Press, Cambridge, UK, 1999.

[9] R. Cornes and T. Sandler, "The Theory of Externalities, Public Goods and Club Goods". Cambridge University Press, Cambridge, UK., 2nd edition, 1996.

[10] R. T. Croson, Partners and strangers revisited. Econ. Letters, 53 (1996), 25-32.

[11] D. D. Davis and C. A. Holt, "Experimental Economics". Princeton University Press, New Jersey, 1993.

[12] J. Dow and S. R. C. Werlang, Risk aversion, uncertainty aversion and the optimal choice of portfolio. Econometrica, 60, 197-204, 1992.

[13] J. Dow and S. R. C. Werlang, Nash equilibrium under uncertainty: Breaking down backward induction. J. of Econ. Theory, 64 (1994), 305-324.

[14] J. Eichberger and D. Kelsey, Uncertainty aversion and preference for randomisation. J. of Econ. Theory, 71 (1996), 31-43.

[15] J. Eichberger and D. Kelsey, E-capacities and the Ellsberg paradox. Theory and Decision, 46 (1999), 107-140.

[16] J. Eichberger and D. Kelsey, Uncertainty and strategic interaction in economics. University of Birmingham discussion paper no. 99-26, 1999. 
[17] J. Eichberger and D. Kelsey, Non-Additive beliefs and strategic equilibria. Games Econ. Behav., 30 (2000), 183-215.

[18] L. G. Epstein, A definition of uncertainty aversion. Rev. Econ. Stud., 66 (1999), 579-606.

[19] P. Ghirardato, On independence for Non-Additive measures and a Fubini theorem. J. Econ. Theory, 73 (1997), 261-291.

[20] P. Ghirardato and M. Marinacci, Ambiguity made precise: A comparative foundation. J. Econ Theory, forthcoming, 1998.

[21] I. Gilboa, Expected utility with purely subjective non-additive probabilities. J. Math. Econ., 16 (1987), 65-88.

[22] M. Gradstein, S. Nitzan, and S. Slutsky, Private provision of public goods under price uncertainty. Soc. Choice Welfare, 10 (1993), 371-382.

[23] S. Hackett, E. Schlager, and J. Walker, The role of communications in resolving commons dilemmas. J. Environ. Econ. and Manage., 27 (1994), 99-126.

[24] R. Isaac, J. M. Walker, and S. H. Thomas, Divergent evidence on free riding: An experimental examination of alternative explanations. Public Choice, 43 (1984), 113-149.

[25] J.van Huyck, R. Battalio, and R. Beil, Tacit coordination games, strategic uncertainty and coordination failure. Amer. Econ. Rev., 80 (1990), 234-248.

[26] D. Kelsey and S. Nandeibam, On the measurement of uncertainty aversion. Technical report, The University of Birmingham, 1996.

[27] D. Kreps, P. Milgrom, J. Roberts, and R. Wilson, Rational cooperation in the finitely repeated prisoner's dilemma. J. Econ. Theory, 27 (1982), 253-279.

[28] J. O. Ledyard, Public goods: A survey of experimental research. In (A. E. Roth, Ed.), "The Handbook of Experimental Economics". Princeton University Press, New Jersey, 1995. 
[29] K. C. Lo, Nash equilibrium without mutual knowledge of rationality. Econ. Theory, 14 (1999), $621-633$.

[30] M. Marinacci, Ambiguous games. Games Econ Behav, 31 (2000), 191-219.

[31] H. Moulin, "Game Theory for the Social Sciences". New York University Press, New York, 1986.

[32] S. Mukerji, Understanding the non-additive probability decision model. Econ Theory, 9 (1997), $23-46$.

[33] J. F. Nash, Two-person cooperative games. Econometrica, 21 (1953), 128-140.

[34] M. J. Ryan, CEU preferences and game theoretic equilibrium. The University of Auckland, working paper series no. 167, 1997.

[35] T. Sandler, F. Sterbenz, and J. Posnett, Free riding and uncertainty. Europ. Econ. Rev., 31 (1987), 1605-1617.

[36] R. Sarin and P. Wakker, A simple axiomatization of non-additive expected utility. Econometrica, 60 (1992), 1255-1272.

[37] D. Schmeidler, Subjective probability and expected utility without additivity. Econometrica, 57 (1989), 571-587.

[38] M. Sefton and R. Steinberg, Reward structures in public good experiments. J. Public Econ., 61 (1996), 263-287.

[39] U. Segal and A. Spivak, First-order versus second-order risk aversion. J. Econ. Theory, 51 (1990), 111-125. 
Endnotes 
Notes

1 
1 It is possible to construct examples when the production function for public goods is exponential, in which public good provision with ambiguity is above the Pareto optimal level.

${ }^{2}$ We could obtain similar results if the word strictly were omitted from this definition. We have not reported these to save space. Note that our definition of strategic complements is slightly stronger than the usual one. The usual definition requires that increasing your own strategy increases the marginal benefit to an opponent of increasing his/her strategy. We require that, in addition, there is a monotonic relationship between your strategy and your opponents' total benefit. The reason for this being that the marginal benefit under ambiguity (see Definition 2.15) depends both on the conventional marginal benefit and on how a player ranks his/her opponents' actions. Thus, by assuming aggregate externalities, we ensure that playing a higher strategy has an unambiguous effect on the marginal incentives of one's opponents'.

${ }^{3}$ We shall assume that all games satisfy concavity because this allows us to state our results in a cleaner way. Similar results could be obtained if pay-off functions were weakly concave.

${ }^{4}$ Epstein [18] has proposed an alternative measure of uncertainty-aversion. We do not have space in the present paper to present a detailed comparison of the two. We would like to note that, for pure equilibria, which we believe to be the more interesting case, we could obtain similar results using Epstein's measure of uncertainty-aversion. Note that [20] and [26] have proposed formal measures of uncertainty-aversion more in the spirit of the present paper.

${ }^{5}$ In [14], we show that individuals with CEU preferences will not have a strict preference for randomisation. This provides a justification for these assumptions.

${ }^{6}$ A symmetric equilibrium is a Nash equilibrium, if beliefs are additive and independent, regardless of the size of the support. We have not required that players' beliefs be independent, since at present there are unresolved questions concerning the definition of independence for capacities, see [17] and [19].

${ }^{7}$ A similar definition has been proposed by Marinacci [30], who built on earlier work by Dow and Werlang [12].

${ }^{8}$ An example of this would be the public goods model with exponential utility.

${ }^{9}$ There are a number of possible extensions to this basic model which one might wish to consider. In particular it has been suggested that the assumptions that utility is linear in the private good and separable between the two goods should be relaxed. In the more general model in section 3, neither of these assumptions is made. As can be seen, most of our conclusions remain valid in that model.

${ }^{10}$ There is also a mixed strategy equilibrium in which all individuals randomise between contributing $m^{*}$ and 0 .

${ }^{11}$ In a subsequent experiment Croson [10] has confirmed the restart effect.

${ }^{12}$ This does not follow directly from the results of section 3, since this model does not satisfy Assumption 3.2 , however it can be established by similar arguments.

${ }^{13}$ This step is valid since, if there are positive or negative aggregate externalities $u\left(m_{1}, s_{-i}\right)$ and $u\left(m_{3}, s_{-i}\right)$ are comonotonic.

\footnotetext{
${ }^{14}$ See Notation A.2.
} 


\begin{abstract}
We examine the effect of ambiguity in symmetric games with aggregate externalities. We find that ambiguity will increase/decrease the equilibrium strategy in games with strategic complements/substitutes and positive externalities. These effects are reversed in games with negative externalities. We consider some economic applications of these results to Cournot oligopoly, bargaining, macroeconomic coordination and voluntary donations to a public good. In particular we show that ambiguity may reduce free-riding. Comparative statics analysis shows that increases in uncertainty will increase donations, to a public good.
\end{abstract}

Keywords: Ambiguity, Strategic Complements, Public Goods, Choquet Integral, Free Rider.

JEL Classification: $\quad$ C72, D81, H41 